\title{
Between Cepheids and the Cosmos
}

\author{
Nancy Remage Evans ${ }^{1, \star}$, Scott Engle ${ }^{2, \star \star}$, Ed Guinan ${ }^{2, \star \star \star}$, Hilding Neilson ${ }^{3, \star \star \star \star}$, Massimo \\ Marengo $^{4, \dagger}$, Lynn Matthews ${ }^{5, \ddagger}$, and Moritz Guenther ${ }^{6, \S}$ \\ ${ }^{1}$ Smithsonian Astrophysical Observatory, 60 Garden St., Cambridge MA 02138, USA \\ ${ }^{2}$ Villanova University, Department of Astronomy, Villanova, PA 19085, USA \\ ${ }^{3}$ University of Toronto, Dept of Astronomy \& Astrophysics, 60 St. George St. Toronto, ON M5S 3H4, Canada \\ ${ }^{4}$ lowa State University, A313E Zaffarano, Dept. of Physics and Astronomy, Ames, IA 50010, USA \\ ${ }^{5}$ MIT Haystack Observatory, Westford MA, 01886, USA \\ ${ }^{6}$ MIT Kavli Institute for Astrophysics and Space Research, 70 Vassar St., Cambridge MA 02139, USA
}

\begin{abstract}
The X-ray observations of three classical Cepheids produce a surprising result. At approximately the phase of maximum radius there is a sharp increase in X-ray flux above the normal "quiescent" level. The relation of this new upper atmosphere diagnostic to other phenonena above the photosphere is discussed.
\end{abstract}

\section{Introduction}

We have a number of tantalizing clues about material above the photosphere in classical Cepheids: spatially extended infrared emission seen with Spitzer, including an apparent bow shock associated with $\delta$ Cephei itself, extended nebulosity detected in the H I 21-cm line, and evidence of circumstellar shells based on near-infrared interferometry ([3]). The understanding of how these are related is patchy. There are a number of photospheric/chromospheric diagnostics from Hubble Space Telescope ultraviolet (UV) spectra, confirming pulsation related activity immediately following minimum radius as discussed in [1]. The recent addition to these diagnostics is a brief X-ray increase at the surprising phase of maximum radius (when the photospheric UV lines are quiescent). This has been seen in two cycles of $\delta \mathrm{Cep}$, and three of $\beta$ Dor. In additon, a number of random phase observations of Cepheids have been made with the XMM-Newton satellite ([2]), summarized in Figure 1. The only Cepheid detected is V473 Lyr, again shortly after maximum radius. The emerging picture is that at the phase when the pulsation wave passes through the photosphere and chromosphere (minimum radius) many features are seen at those levels - UV emission lines and an increase in microturbulence. There is, however, no increase in X-rays flux above the level typically produced in a star with a convective envelope ("quiescent" flux). However, at maximum radius as the star starts to contract the X-ray

\footnotetext{
^nevans@cfa.harvard.edu

$\star \star$ scott.engle@villanova.edu

$\star \star \star$ edward.guinan@villanova.edu

$\star \star \star \star$ neilson@astro.utoronto.ca

†mmarengo@iastate.edu

¥lmatthew@haystack.mit.edu

§hgunther@mit.edu
} 


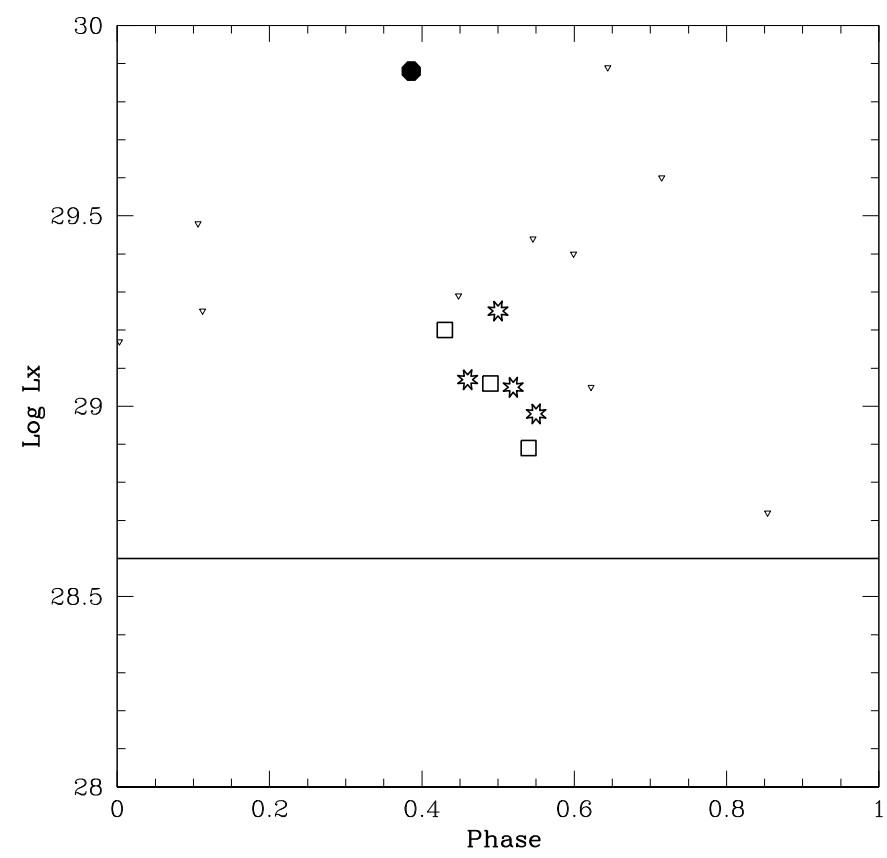

Figure 1. Random phase XMM observations of Cepheids, compared with observations of $\delta \mathrm{Cep}$ (asterisks) and $\beta$ Dor (squares). Upper limits (triangles) were found for all Cepheids in the XMM observations, except V473 Lyr (filled circle), which was observed at maximum radius, the same phase as the increased flux in $\delta$ Cep. The horizontal line is the quiescent level of X-rays for $\delta$ Cep from 9 observations at other phases. $L_{X}$ is in ergs $\sec ^{-1}$.

flux increases dramatically by nearly a factor of five. In contrast, photospheric and chromospheric indicators are at a low level in this "coasting phase". The morphology of the X-ray increase is a rapid rise, a short duration and a rapid fall, reminiscent of a flare or a pulsation driven shock. Thus, the "working model" is that the X-ray activity occurs above the photosphere and chromosphere, and something triggers the X-ray event as the atmosphere starts to collapse. Importantly, this ties the $\mathrm{X}$-ray increase to the pulsation process.

Are the X-rays (at about $0.1 \mathrm{AU}$ ) related to the circumstellar envelopes (CSEs, at about $1 \mathrm{AU}$ ) and the possible bow shock seen in $\delta$ Cep (at $10^{4}$ to $10^{5} \mathrm{AU}$ )? One possible scenario would be for the atmospheric collapse to trigger a coronal loop reconnection event. This might in turn result in a coronal mass ejection event. Altogether this might produce mild outflow similar to the solar wind. If this scenario proves correct, it would have important implications for mass loss in Cepheids (related to evolutionary calculations) as well as the influence of the CSE on the Cepheid Leavitt law (periodluminosity relation).

Acknowledgments: Support was provided by Chandra X-ray Center NASA Contract NAS8-03060.

\section{References}

[1] Engle, S., Guinan, E. F., Harper, G. M., Neilson, H. R., \& Evans, N. R., ApJ, 794, 80 (2014)

[2] Evans, N. R., Pillitteri, I., Wolk, S., et al., AJ, 151, 108 (2016)

[3] Marengo, M., Evans, N. R., Matthews, L. D. et al., ASSP, 31, 99 (2013) 